die geltende Verfassungsordnung angreifen, bekämpfen und diffamieren. Sie gingen

über den Rahmen theoretisch-wissenschaftlicher Erkenntnisse hinaus.

$[\ldots]$

Die Bemerkung über die Sinnlosigkeit des Auswechselns der Personen aus dem »korrupten « und dem »demokratiefeindlichen « Parteiapparat läßt erkennen, daß nicht einzelne Maßnahmen des Senats von Berlin im Zusammenhang mit den Ereignissen der Jahre 1967 und 1968 kritisiert wurden, sondern daß es dem Beigeladenen darum ging, die Institutionen als solche abzuschaffen, weil er sie, was sich auch im Zusammenhang mit seinen Darlegungen zu seinem Demokratieverständnis ergibt, für undemokratisch hielt.

$[\ldots]$

[Az.: VB 49, 73]

gez. Bergmann

Bartelt

Schröder

\title{
Anmerkungen:
}

\section{A. Staatsphilosophie - eine bisher unbekannte Form der Innenpolitik}

Es gibt eine effiziente und kostensparende Methode, die aufwendigen Kontroversen zwischen Wissenschaftlern zu beenden: die Staatsdoktrin. Jahrzehnte, ja jahrhundertelang schien diese Methode vergessen, schien niemand mehr sich daran zu erinnern, daß man Theorien sei's anordnen, sei's verbieten kann, wie ehedem Galileis Kosmologie, aber in jüngster Zeit hat man in einem neuerwachten Geschichtsbewußtsein zu der verschütteten Tradition zurückgefunden. Die gewählten Volksvertreter haben begonnen, sich wissenschaftstheoretisch zu betätigen und zu bestimmen, was eine wissenschaftliche Theorie ist und was nicht. Wo Philosophen endlos stritten und Berge von Büchern füllten, um zu definieren, wie das Verhältnis von Theorie und Praxis aussieht, was eine wissenschaftlich erwiesene Wahrheit ist, was unter einem rationalen Beweis verstanden werden muß, ohne zu einem endgültigen und von allen geteilten Resultat gelangen zu können, da greifen heute die Politiker ein. Das gilt zumindest für die Bundesrepublik, die diese avantgardistische Entwicklung in der westlichen Welt anführt und damit auf das Wirtschaftswunder ein Wissenschaftswunder folgen läßt. Ihre Staatsorgane haben entdeckt, daß Poppers Falsifikationstheorie die einzig wissenschaftliche Wissenschaftstheorie ist. Sie bestätigen damit, daß wir hierzulande ein Volk von Dichtern und Denkern sind und daß wir den Traum von Platon haben Wirklichkeit werden lassen: unsere Politiker sind Philosophen.

Wir haben eine philosophische Sozialdemokratie, eine philosophische CDU, philosophisch orientierte Gerichte, wie die neuere Rechtsprechung zeigt, und eine philosophische Bildzeitung, genauer, eine philosophische BZ. Es gibt in der Bundesrepublik gleichsam eine große philosophische Koalition, und was das Verblüffendste ist, diese Koalition beruht nicht auf Kompromiß, sondern auf wahrhafter

\footnotetext{
* Den folgenden Anmerkungen liegen zwei Referate zugrunde, die am 27. Januar 1977 in der Freien Universität in West-Berlin auf einer vom Fachbereich für Politische Wissenschaft und vom Zentralinstitut für Sozialwissenschaftliche Forschung der FU-Berlin zum Thema »Wissenschaft und Recht und Freiheit« abgehaltenen Veranstaltung vorgetragen wurden. (Red. KJ).
} 
Ubereinstimmung: die staatstragenden Organe fußen in ihrem philosophischen Bekenntnis allesamt auf Poppers Kritischem Rationalismus'. Was zu zeigen ist. Die philosophische Aktivität der staatlichen und halbstaatlichen Instanzen in der Bundesrepublik nahm ihren Anfang, als Mitte der sechziger Jahre die innerwissenschaftliche Diskussion über wissenschaftstheoretische Fragen wieder in Gang kam und Alternativen, auch marxistische, wieder diskutierbar wurden ${ }^{2}$. Die sicher geglaubte Trennung zwischen Theorie und Praxis, zwischen Wissenschaft und Ethik, zwischen Erfahrungswissen und Werturteil wurde neu reflektiert, geriet partiell ins Wanken und damit auch das Grundaxiom der Konzeption von Wissenschaft, wie es von der Popperschule vertreten wird. Die ansonsten wenig theoretische, aber volksnahe BZ ahnte als erste die Bedeutung der Entwicklung und schrieb bereits 1967, als während des Notstandshearings das Verhältnis zwischen Wissenschaftlern und Politikern problematisch zu werden drohte:

"Was diese Aussprache mit aller Deutlichkeit gezeigt hat ist dies: Wissenschaft und Politik sind zweierlei. In so schwerwiegenden politischen Fragen wie gerade die der Notstandsgesetzgebung können Wissenschaftler zwar wertvolle Hinweise und dem Abgeordneten Entscheidungshilfe geben, aber:... Die Entscheidung bleibt beim Politiker und seinem Gewissen. $\alpha^{3}$

Die freie Presse, Rückgrat einer demokratischen Gesellschaft, hatte damit in Erinnerung gerufen, was modische Diskutiersucht hatte vergessen lassen: Wissenschaft und Politik gehören getrennten Sphären an, sind »zweierlei«, und das heißt, politische Entscheidungen haben sich nicht vor dem fragwürdigen und endlos diskutierenden Forum der wissenschaftlichen Erkenntnis zu legitimieren, sondern einzig und allein vor dem kristallklaren Gewissen des Politikers ${ }^{4}$. Allerdings, das klare Bekenntnis zum politischen Kernpunkt der Popperschen Wissenschaftstheorie seitens der BZ war und blieb lange Zeit eine wegweisende Einzelleistung. Die Konzeption der Popperschule wurde in Fachkreisen zwar ausführlich zur Kenntnis genommen und diskutiert, sie war jedoch weit davon entfernt, in ihrer Evidenz unter den Fachwissenschaftlern allgemeine Anerkennung zu finden und insbesondere von den Parteien und der Justiz als einzig sinnvolle Theorie der wissenschaftlichen Erkenntnis gewürdigt und institutionell durchgesetzt zu werden. Einer solchen Durchsetzung entsprach weder das allgemeine außerwissenschaftliche Bewußtsein, das in wissenschaftstheoretischen Spezialfragen eher zur Indifferenz neigte, noch die damals vorherrschende relativ wörtliche Interpretation des Grundgesetzes, das ja in Artikel s die Wissenschaft als frei deklariert und damit die Entscheidung über die Stichhaltigkeit oder die Fragwürdigkeit einer Theorie ausdrücklich zu einer rein wissenschaftlichen Entscheidung macht. Noch 1973 urteilte das Bundesverfassungsgericht im "Hochschulurteil «:

„Das in Art. 5 Abs. 3 GG enthaltene Freiheitsrecht schützt als Abwehrrecht die wissenschaftliche Betätigung gegen staatliche Eingriffe und steht jedem zu, der wissenschaftlich tätig ist oder tätig werden will. Dieser Freiraum des Wissenschaftlers ist grundsätzlich ... vorbehaltlos

I Vor allem entwickelt in: Popper, Karl R.: Logik der Forschung, 1934; 4. verb. Aufl. Tübingen 1971; sowie in: Conjectures and Refutations, London 1963.

$2 \mathrm{Vgl}$. insb. die sich an die Tübinger Arbeitstagung der Deutschen Gesellschaft für Soziologie (196I) anschließende Kontroverse zwischen J. Habermas u. H. Albert, abgedruckt in: Theodor W. Adorno u. a. (Hrsg.): Der Positivismusstreit in der deutschen Soziologie, Darmstadt u. Neuwied 1972.

3 BZ vom 10. Nov. 1967; der Kommentar trug übrigens den Titel "Notstand der Professoren «.

4 Kleine erfreuliche Anmerkung zur Problematik der Gewissenspolitik: Der Münchener SPD-Abgeordnete Rudolf Schöffberger empfahl in seiner Auseinandersetzung mit dem Münchener Oberbürgermeister als Konsequenz der Gewissenslehre, Politiker, die sich in ihren Entscheidungen auf ihr Gewissen zu berufen pflegen, statt auf die Partei, in deren Namen sie sprechen, sollten sich bei der nächsten Wahl dann auch von diesem Gewissen aufstellen lassen ...; wörtl. in: R. Schöffberger: Imperatives Mandat und innerparteiliche Demokratie, in: Rolf Seliger (Hrsg.): Konzepte, SPD 74, Kritische Beiträge zur Mobilisierung der Sozialdemokratie, München 1974 , S. I $8 \mathrm{f}$. 
geschützt ... In ihm herrscht absolute Freiheit vor jeder Ingerenz öffentlicher Gewalt. In diesen Freiraum fallen vor allem die auf wissenschaftlicher Eigengesetzlichkeit beruhenden Prozesse, Verhaltensweisen und Entscheidungen bei dem Auffinden von Erkenntnissen, ihrer Deutung und Weitergabe."

Und das Gericht fährt bekräftigend fort:

"Damit sich Forschung und Lehre ungehindert an dem Bemühen um Wahrheit als retwas noch nicht ganz Gefundenes und nie ganz Aufzufindendes. (Wilhelm von Humboldt) ausrichten können, ist die Wissenschaft zu einem von staatlicher Fremdbestimmung freien Bereich ... erklärt worden. Damit ist zugleich gesagt, daß Art. 5 Abs. 3 GG nicht eine bestimmte Auffassung von der Wissenschaft oder eine bestimmte Wissenschaftstheorie schützen will.«s

Das Urteil dokumentiert deutlich, daß der Gedanke, daß wissenschaftstheoretische Kontroversen politisch entschieden werden können, daß es möglich und sinnvoll und zugleich vom Grundgesetz gedeckt ist, «eine bestimmte Auffassung von der Wissenschaft oder eine bestimmte Wissenschaftstheorie « institutionell durchzusetzen, in seiner Bedeutung und Zweckmäßigkeit noch nicht erfaßt war. Umständlich ist die Rede von "Fremdbestimmung « und »wissenschaftlicher Eigengesetzlichkeit« und das heißt: die vom Grundgesetz geschützte Freiheit der Wissenschaft wurde noch immer negativ als »Abwehrrecht . . gegen staatliche Eingriffe «, denn im Sinne von staatlicher Mitwirkung interpretiert. Das hat sich jedoch geändert. Mit seinem Urteil vom Juni 1976 hat das Oberverwaltungsgericht Berlin mit großem philosophischen Selbstbewußtsein die Trennung von Wissenschaft und Politik juristisch verordnet und damit amtlich klargestellt, daß - seiner Rechtsauffassung nach - die wissenschaftstheoretische Kontroverse über das Theorie-Praxis-Problem im Geltungsbereich des Grundgesetzes nunmehr als beendet angesehen werden sollte. Die in Fachkreisen bislang immer noch unklare Grenze zieht das Gericht unter Berufung auf die freiheitlich-demokratische Grundordnung wie folgt:

"Die Wissenschaft als solche kann ... niemals gegen die freiheitlich-demokratische Grundordnung verstoßen, auch wenn sie zu einer Prognose künftiger gesellschaftlicher Entwicklungen führt. Der durch Art. S Abs. 3 GG geschützte Freiheitsraum endet aber dort, wo wissenschaftliche Erkenntnisse in die politische Wirklichkeit umgesetzt werden sollen und zu politischem Handeln aufgerufen wird. Die Grenze zwischen wissenschaftlicher Theorie und politischem Ziel liegt dort, wo die betrachtend gewonnenen Erkenntnisse zu Bestimmungsgründen politischen Handelns gemacht werden.«

War im zuvor zitierten Urteil bestimmend die Rede von »Freiraum«, »Freiheitsrecht ... als Abwehrrecht«, "absoluter Freiheit« und "vorbehaltlos geschützt «, so liegt hier der Akzent auf »endet«, »Grenze» und »aber«. Das Gericht fährt präzisierend fort:

"Für Wissenschaftler in politikbezogenen Disziplinen ... mag sich ein Eingehen auf tagespolitische Fragen im Rahmen des Art. 5 Abs. 3 GG halten, wenn es in sachlicher Form und sorgsamem Abwägen des Für und Wider geschieht. Sobald ein solcher Wissenschaftler die politische Aktivität des Hörers oder Lesers anspricht - gleichgültig, ob innerhalb oder außerhalb von Lehrveranstaltungen - oder sich die Äußerungen in politischer Propaganda verlieren, ist der Freiheitsraum Art. 5 Abs. 3 GG überschritten. $\aleph^{6}$

\footnotetext{
5 Urteil vom 25. 5. 1973, in: Entscheidungen des Bundesverfassungsgerichts, 35. Band, Tübingen 1974, S. I $12 \mathrm{f}$

6 Urteil vom 10. 6. 1976, oben S. 76 ff.

Interessant ist der politische Hintergrund der Urteilsbegründung; die entscheidenden Sätze sind nahezu wörtlich dem berühmten KPD-Urteil aus der Zeit des Kalten Krieges entnommen, ohne als solche gekennzeichnet zu sein. Im KPD-Urteil lautet die entsprechende Passage:

"Sie (die Wissenschaft) kann, auch wenn sie zu einer Prognose künftiger Entwicklungen führt, als solche niemals gegen die freiheitliche demokratische Grundordnung verstoßen... Die eindeutig bestimmbare Grenze zwischen wissenschaftlicher Theorie und politischem Ziel liegt dort, wo die betrachtend gewonnenen Erkenntnisse von einer politischen Partei, also einer ihrem Wesen nach zu aktivem Handeln im staatlichen Leben entschlossenen Gruppe, in ihren Willen aufgenommen, zu
} 
Das sorgsame Abwägen des Für und Wider - gewiß, dies ist die Aufgabe der Wissenschaft. Aber noch einmal: Sobald "wissenschaftliche Erkenntnisse in die politische Wirklichkeit umgesetzt werden sollen«, sobald "die betrachtend gewonnenen Erkenntnisse zu Bestimmungsgründen politischen Handelns gemacht werden «, »sobald ein... Wissenschaftler die politische Aktivität des Hörers oder Lesers anspricht", ist der Boden der Wissenschaft verlassen. Die unpolitische Wissenschaft als Staatsdoktrin? Als hätte es nie ein Naziregime und das Problem beschämend passiver Fachexperten gegeben. Als hätte niemals eine Debatte darüber stattgefunden, ob nicht jene Wissenschaftler, die mit ihrer engagierten Lehre zweifellos die politische Aktivität ihrer Hörer ansprachen und darum in die Emigration gezwungen wurden, ob nicht sie der Idee der Wissenschaft besser gedient haben als jene sich selbst als apolitisch mißverstehenden Fachgelehrten, deren Arbeit ob ihrer sonderbaren Indifferenz mit den herrschenden Verhältnissen niemals in Konflikt geriet... Doch zurück zu unserer positiven Würdigung der laufenden philosophischen Entwicklung.

Mit dem Urteil des Oberverwaltungsgerichts Berlin hat sich die wissenschaftstheoretische Diskussion endlich dahin verlagert, wo sie von alters her hingehört: vor die Justiz. Der Richterspruch legt definitiv für jeden einzelnen Forscher klar, was Wissenschaft im Sinne der freiheitlich-demokratischen Grundordnung ist oder sein soll, gibt dem noch Unsicheren eine feste Leitlinie und erlaubt es zugleich, noch vorhandene Abweichler amtlich zu belehren oder - falls unbeirrbar - aus dem Bereich der Institution Wissenschaft zu entfernen. Das Urteil ist gleichsam eine Konkretisierung der Radikalenentscheidung für den Bereich der Wissenschaft: es entlastet zumindest vorübergehend von inhaltlichen Debatten und könnte damit der Gelehrtenrepublik einen ähnlich gravierenden Fortschritt bescheren wie der Richterspruch vom Mai 1975 für die Bundesrepublik insgesamt ${ }^{7}$.

Doch woher die Einsicht des Gerichts? Wieso konnte die Philosophie, gemäß einer alten marxistischen Forderung, hier endlich praktisch werden? Zweifellos wäre die beschriebene Entwicklung nicht möglich gewesen ohne das philosophische Engagement von außerjuristischen politischen Instanzen und ohne die tätige Mithilfe von praktisch gesonnenen Männern aus den Reihen der Wissenschaftler selbst. Bereits 197 I legte Kurt Sontheimer die Grundlinien für eine Art von freiheitlich-demokratische Grundwissenschaft fest. In einem Rundfunkvortrag deutete er an, daß die Wissenschaft zwar eine Vielzahl von Methoden braucht, aber beileibe nicht alle und schon gar nicht jene, die sich selbst als marxistisch verstehen und wies damit weitsichtig auf die generelle Möglichkeit einer Effektivierung des Wissenschaftsbetriebes durch Beschränkung und rechtsförmigen Ausschluß hin:

"Der auf der Idee freier Wissenschaft beruhende Wissenschaftspluralismus setzt . . einen Konsensus voraus, nämlich die Ưbereinstimmung darüber, daß es legitim ist, mit verschiedenen Methoden und von verschiedenen weltanschaulichen Prämissen her die Wirklichkeit zu erkennen und der Wahrheit näherzukommen. Die marxistische Gegenposition innerhalb des wissenschaftlichen Pluralismus ist - so meine ich - vergleichbar den verfassungsfeindlichen Parteien, deren Programmatik und Verhalten den Prinzipien der pluralistischen Demokratie widerspricht. ${ }^{8}$

Bestimmungsgründen ihres politischen Handelns gemacht werden. In: Entscheidungen des Bundesverfassungsgerichts, 5. Band, Tübingen 1956, S. 146 (Urteil vom 17. 8. 1956).

Die entscheidende Differenz: Im KPD-Urteil wird die »Grenze zwischen wissenschaftlicher Theorie und politischem Ziel * erst überschritten, wenn die Erkenntnisse "von einer politischen Partei zu "Bestimmungsgründen ibres politischen Handelns" gemacht werden. Zwanzig Jahre später fällt der Parteienvorbehalt weg; die Theorie-Praxis-Grenze wird für den je einzelnen Wissenschaftler verbindlich minnerhalb oder außerhalb von Lehrveranstaltungen « - d. h. für die Forschung schlechthin.

7 "Radikalentscheidung ", siehe den Beschluß vom 22. 5. 1975, in: Entscheidungen des Bundesverfassungsgerichts, 39. Band, Tübingen 1975 , S. $334 \mathrm{ff}$.

8 Kurt Sontheimer: Vortrag über Wissenschaftspluralismus im Hessischen Rundfunk, 1971; zit. in: 
Soweit Kurt Sontheimer, dessen theoretische Klarheit - so kann man zumindest rückblickend feststellen - die nunmehr gültige Praxis frappierend weitsichtig vorwegnahm.

Doch ich sprach eingangs von der großen philosophischen Koalition und deutete an, daß das philosophische Erwachen auch in den großen Parteien der Bundesrepublik eingesetzt hat. In der Tat, was die philosophischen Grundlagen von Wissenschaft und Politik anbelangt, herrscht in den staatstragenden Organisationen seit geraumer Zeit eine wundersame Harmonie; die führenden Staatsmänner haben nicht gesäumt, sich in die schwierige Fachmaterie einzuarbeiten, ihre gewonnenen Einsichten in das richtige und wünschenswerte Verhältnis von Wissenschaft und Politik den bis dato immer noch zerstrittenen Fachleuten wegweisend kundzutun und - auf die Gefahr hin, als Hobby-Philosophen verkannt zu werden - auch publizistisch hervorzutreten.

Von seiten der SPD wurde 1975 als erstes Resultat der staatsmännischen Theoriearbeit ein vielbeachtetes Buch mit dem Titel »Kritischer Rationalismus und Sozialdemokratie « vorgelegt ${ }^{9}$, eingeleitet mit einem Vorwort von Kanzler Helmut Schmidt. Der Kanzler bekannte zwar erschöpft:

»Man wird von einem, der an Verantwortung für Staat und Partei schon genug trägt, nicht erwarten, daß er sich außerdem den politischen Schulmeister in Sachen Philosophie antragen läßt.«

Dennoch nahm er die ihm angetragene Zusatzaufgabe wahr und formulierte - die Trennung von politisch-moralischer Handlung und theoretisch-rationaler Erkenntnis hervorhebend - verantwortungsbewußt ganz im Sinne Poppers:

»Der politische Wert einer 'Theorie besteht in der Dienstleistung, dem politisch Handelnden die tatsächlichen Möglichkeiten zum Handeln aufzuzeigen und die vorhersehbaren Folgen verschiedener Handlungsweisen. Der politisch Handelnde bleibt aber belastet mit einem großen Rest von Ungewißheit. Vor allem aber bleibt er belastet mit der Verantwortung für die Werturteile, die nur sittlich zu begründen sind... Keine ,Theorie kann ihm das Werturteil abnehmen. « ${ }^{10}$

Der Spiegel kommentierte damals pointiert und zutreffend:

"Sir Karl Popper, der in Wien geborene und in England lebende bedeutendste Wissenschaftsphilosoph der Gegenwart, ... wird ein Leitbild für Sozialdemokraten.

Nicht mehr Hegel und Marx, und damit der Enthusiasmus einer prophetischen Geschichtsphilosophie, sind ... Wegweiser der Partei, sondern Kant und Popper .. . Schmidt betont: 'Kants Nüchternheit und Klarheit im Denken wird heute von den kritischen Rationalisten geteilt. Mit diesem Bekenntnis eröffnet der Bundeskanzler eine neue Phase in der Geschichte der Sozialdemokratie.«"

Diesem Kommentar ist nichts weiter hinzuzufügen als das gewiß nicht unbedeutende Faktum, daß die Sozialdemokratie in ihrem philosophischen Engagement - wie in so vielen ihrer staatsfördernden Aktivitäten - von den Politikern der christdemokratischen und christlich-sozialen Parteien weit übertroffen wird. Nicht was den Inhalt der Lehre anbelangt, wohl aber in der Konsequenz, mit der sie vertreten und durchgesetzt wird. Jüngst wurde von den Kultusministern der CDU/CSU-regierten

Horst Heinemann: Die Kontroverse um den Wissenschaftspluralismus, in: Beilage zur Wochenzeitung "Das Parlament «, B 26/74, S. 8 .

9 G. Lührs, T. Sarrazin, F. Spreer, M. Tietzel (Hrsg.): Kritischer Rationalismus und Sozialdemokratie, Berlin/Bonn-Bad Godesberg 1975.

10 Ebda., S. XI u. XIV.

I Der Spiegel, Nr. 19/1975, S. 167 f. Anzumerken ist noch, daß Schmidt sich trotz seines inhalt lichen Bekenntnisses zu Kant und Popper selber nicht als Anhänger des Kritischen Rationalismus begreift und theoretischen Alternativen verbal Raum gewährt. Schmidt schreibt: „Ich bin kein Marxist, ich bin ebensowenig ein Anhänger des kritischen Rationalismus. Jedoch empfehle ich, Marx zu lesen, ebenso Popper... und viele andere - Kant bitte nicht zu vergessen ..." (Vorwort, a. a. O., S. XV). 
Länder eine Rahmenrichtlinie für »Politische Bildung « in Auftrag gegeben (Untertitel: "Grundlagen und Zielprojektionen für den Unterricht an Schulen«), aus der ersichtlich wird, daß der philosophische Klärungsprozeß innerhalb der CDU bereits weitgehend abgeschlossen ist. Er ist im Begriffe, institutionelle Konsequenzen von großer Tragweite zu zeitigen: Künftig soll nach den Plänen von CDU und CSU an den Schulen, den wichtigsten Bildungsinstitutionen des Landes, nur noch nach Methoden und Ansätzen unterrichtet werden, die den Wissenschaftskriterien des Kritischen Rationalismus entsprechen. ${ }^{2}$ Unter der Uberschrift "Wissenschaftsbezug « heißt es in der Schrift:

"Auch in der politischen Bildung muß ... mit verschiedenen Theorieansätzen und Methoden gearbeitet werden. Diese Pluralität findet ihre Grenze dort, wo Mindestanforderungen der Wissenschaftlichkeit nicht gewahrt werden.

Dazu gehören: 1 . Aussagen, die den Anspruch auf Wissenschaftlichkeit erheben, müssen intersubjektiv nachprüfbar sein. Diese Bedingung ist nur erfüllt, wenn Wertprämissen, Kriterien der Faktenauswahl und Methoden der Faktenverarbeitung offengelegt werden; Aussagen präzise und möglichst eindeutig formuliert werden; Aussagen prinzipiell widerlegbar sind, d. h. sie dürfen sich nicht aus logischen Gründen der Nachprüfbarkeit entziehen. «'s

Poppers Falsifikationismus als Richtlinie der deutschen Schulphilosophie? Mit dieser Planung, sofern sie aufgeht, dürfte die Zeit der verwirrenden Methodenvielfalt dem Ende zugehen. Denn die formulierten "Mindestanforderungen der Wissenschaftlichkeit " sind nicht die Anforderungen der Wissenschaft schlechthin, sondern die der Popperschen Auffassung von Wissenschaft; und die damit explizit vorgenommene Gleichsetzung von wissenschaftlicher Methodik und Popperianismus ist kein Fehler, sondern bewußt intendiert: Ausdrücklich erklären die CDU-Minister in ihrem gemeinsam unterzeichneten Vorwort, daß sich ihr Programm als

"Alternative zu jenen Ansätzen, die der Kritischen Theorie verhaftet bleiben« begreift, und daß es "zur Orientierung von Lernzielen und Lerninhalten an der Verfassung « beitragen soll. ${ }^{14}$

Die Theoretiker der Frankfurter Schule, ehedem in die Emigration verbannt und nach dem Kriege ehrerbietig in die Bundesrepublik zurückgebeten - sie werden abermals aus dem Bereich der als legitim angesehenen Wissenschaft hinausgedrängt? Irrtum, man drängt sie nicht hinaus, sie werden hinausdefiniert, und mit ihnen alles, was sich den Kriterien des Kritischen Rationalismus nicht fügt. Das Ende der Entwicklung, wie die CDU sie vor Augen hat, wäre die Realisierung einer exklusiven wissenschaftlichen Einheitsmethodik, wäre Freiheit nicht »der«, sondern »einer" Wissenschaft, wäre der Kritische Rationalismus als Staatsdoktrin's.

I2 Vgl. D. Grosser, M. Hättich, H. Oberreuter, B. Sutor: Politische Bildung, Grundlagen und Zielprojek tionen für den Unterricht an Schulen, hrsg. u. mit einem Vorwort vers, von W. Braun, W. Hahn, H. Maier, W. Remmers, W. Scherer, B. Vogel, Stuttgart 1976.

13 Ebda., S. 23. Poppers entsprechende Definition lautet: "Insofern sich die Sätze einer Wissenschaft auf die Wirklichkeit beziehen, müssen sie falsifizierbar sein, und insofern sie nicht falsifizierbar sind beziehen sie sich nicht auf die Wirklichkeit «. Vgl. Logik der Forschung . .., S. 256 (im Original kursiv). Diese Definition aus Poppers Hauptwerk wird in abgewandelten Formulierungen in fast allen seinen Veröffentlichungen wiederholt; in dt. Sprache zuletzt im oben zit. Buch "Krit. Rationalism. u. Sozialdemokratie *. Dort heißt es: " . . eine Theorie gehört dann zur Wissenschaft, wenn sie prinzipiell widerlegbar ist." Vgl. Bryan Magee: Kritischer Rationalismus - Eine Unterhaltung mit Karl Popper, a. a. O., S. 67.

I4 Politische Bildung... (Anm. I 2), S. I.

Is Kommentar der Frankfurter Rundschau: "Der Vorgang ist einzigartig: Fünf Kultusminister der CDU/CSU lassen sich von vier CDU-Politikwissenschaftlern Grundlagen und Ziele für die Politische Bildung in den von ihnen regierten Ländern und den Schulen schreiben... Vorwort und Papier unterstellen einen Ausschließlichkeitsanspruch: Wer nicht dafür ist, steht nicht auf dem Boden der Verfassung... Wenn die CDU-Kultusminister trotzdem meinen, ihr Papier müsse von allen, die auf dem Boden der Verfassung stehen, als gemeinsame Basis Anerkennung finden, dann machen sie eine Parteiideologie, ihre Parteiideologie, zur verbindlichen Richtlinie für Politik und politische Bildung. Das ist: Gleichschaltung und Indoktrination." FR vom 25. 5. $1976 .-$ Kritisch zum Gesamtkomplex erstmals Margherita v. Brentano: Wissenschaftspluralismus - zur Funktion, Genese und Kritik eines 
Es war hier nicht der Ort, auf den Inhalt der Theorie des Kritischen Rationalismus einzugehen. Ich teile die Konzeption Poppers nicht, kann aber versichern, daß Poppers Forschungen - abgesehen von ihrem politisch-polemischen Gehalt - einen entscheidenden, wenn auch kritisch zu reflektierenden Beitrag zur Weiterentwicklung einer der neuzeitlichen Wissenschaft angemessenen Erkenntnistheorie darstellen. ${ }^{16}$

Bemerkenswert ist allerdings, daß unsere Politiker und Hobby-Philosophen die Lehre Poppers just zu dem Zeitpunkt entdecken und zu verbreiten beginnen, da sie von einigen der gegenwärtig prominentesten Wissenschaftsforscher der Gegenwart verworfen wird, ja widerlegt worden ist, da also die wissenschaftstheoretische Diskussion wieder neu beginnt und dabei ist, eine neue und relativ unerwartete Richtung einzuschlagen. Ich denke hier an die amerikanische science of science, die sich unter dem Namen Wissenschaftswissenschaft und Wissenschaftssoziologie auch bei uns als dominierende erkenntnistheoretische Forschungsrichtung zu etablieren beginnt; und ich denke an die Studien von Thomas S. Kuhn ${ }^{17}$, der aufgrund umfassender empirischer Analysen hat zeigen können, daß die Wissenschaftsentwicklung keineswegs, wie Popper behauptete, vom Prinzip permanenter Widerlegungsversuche getragen ist, sondern daß sie bestimmt ist von den Normen, die den Wissenschaftlern (Kuhn: der "scientific community «) von außerwissenschaftlichen, d. h. gesellschaftlichen Instanzen und Umständen auferlegt sind.

Das bedeutet natürlich nicht, daß die Theorie des Kritischen Rationalismus nunmehr nicht mehr vertreten würde, daß sie - weil empirisch falsifiziert - aufgehört habe zu existieren; sie findet nach wie vor ihre Anhänger. Das bedeutet aber allerdings, daß sie eine entscheidende Relativierung und kritische Revision erfuhr ${ }^{18}$, so daß Paul K. Feyerabend - ursprünglich ebenfalls vom Kritischen Rationalismus kommend und neben Kuhn und Popper der wohl bekannteste Wissenschaftstheoretiker der Gegenwart - sagen kann: das Sonderbare an der Falsifikationstheorie ist, daß sie, obwohl falsifiziert, von ihren Anhängern fröhlich weiterpraktiziert wird und sich damit selbst ad absurdum führt.'

Kampfbegriffs, in: Das Argument, Nr. 66/197ı, S. 476 ff.; siehe auch Ulrich K. Preuß: Wissenschaftspluralismus und Verfassungsschutz, in: Legalität und Pluralismus, Frankfurt 1973, S. i is ff.

16 Allgemein zur neueren erkenntnistheoretischen Problematik siehe meine Schrift: Gesellschaftsform und Erkenntnisform; zum Zusammenhang von wissenschaftlicher Erfahrung und gesellschaftlicher Entwicklung, Frankfurt/New York 1976.

17 Insb. in: Die Struktur wissenschaftlicher Revolutionen, 1962; deutsch Frankfurt 1967 (Taschenbuchausg. 1973).

18 Popper über Kuhn: "Die Kritik, die Professor Kuhn meinen Ansichten über die Wissenschaft zukommen ließ, ist eine der interessantesten, denen ich bisher begegnet bin.« Vgl.: Die Normalwissenschaft und ihre Gefahren, in: Kritik und Erkenntnisfortschritt, Abhandlungen des internationalen Kolloquiums über die Philosophie der Wissenschaft, London I965; deutsch Braunschweig 1974, S. 51.

19 Feyerabend: »... Was soll man dann von der methodologischen Forderung halten, daß eine Theorie an der Erfahrung beurteilt werden soll, und daß sie verworfen werden muß, wenn sie anerkannten Basissätzen widerspricht? Was soll man von den verschiedenen Theorien der Bestätigung und Bewährung halten, die alle von der Voraussetzung ausgehen, daß Theorien zur vollständigen Übereinstimmung mit den bekannten Tatsachen gebracht werden können, und die den Grad der erreichten Ubereinstimmung zum Maßstab ihrer Beurteilung machen? Diese Forderung, diese Theorien entpuppen sich jetzt alle als völlig unbrauchbar... In der Praxis hält sich überhaupt niemand an sie. Die Methodologen mögen auf die Bedeutung von Falsifikationen hinweisen - aber sie verwenden fröhlich falsifizierte Theorien .... ; siehe: Wider den Methodenzwang, 1975; deutsch Frankfurt 1976, S. 102 f. Und explizit S. 249: "Fassen wir zusammen: wohin man auch blickt, welche Beispiele man auch betrachtet, es zeigt sich, daß die Grundsätze des kritischen Rationalismus ... eine unrichtige Darstellung der Entwicklung der Wissenschaft in der Vergangenheit geben und die Wissenschaft in der Zukunft nur behindern können. . 